\title{
A Hybrid Method For Automatic Counting OF MICROORGANISMS IN MICROSCOPIC IMAGES
}

\author{
P.Kalavathi and S.Naganandhini \\ Department of Computer Science and Applications \\ Gandhigram Rural Institute-Deemed University \\ Gandhigram, Tamil Nadu, India
}

\begin{abstract}
Microscopic image analysis is an essential process to enable the automatic enumeration and quantitative analysis of microbial images. There are several system are available for numerating microbial growth. Some of the existing method may be inefficient to accurately count the overlapped microorganisms. Therefore, in this paper we proposed an efficient method for automatic segmentation and counting of microorganisms in microscopic images. This method uses a hybrid approach based on morphological operation, active contour model and counting by region labelling process. The colony count value obtained by this proposed method is compared with the manual count and the count value obtained from the existing method.
\end{abstract}

\section{KEYWORDS}

Microscopic images, Active contour method, CV algorithm, Morphological operation, Automatic microbial counting.

\section{INTRODUCTION}

Microbes are tiny organism mostly single-celled and rarely multiple-celled. These organism are exist in free living or symbiotically associations with plants and animals as well as in the human body. Microbes are classified into various groups such as: Bacteria, Fungi, Protozoa, Algae and Viruses. A typical Bacteria are single-celled organism and they multiply once in every 20 minutes. Some of the bacteria are harmless called non-pathogenic and some are harmful called pathogenic i.e., over $70 \%$ of bacteria are non-pathogenic and the remaining are pathogenic. Bacteria are classified into four major groups based on their cell wall characteristics [1], which includes Gram positive, Gram negative, Cell wall less bacteria (microplasmas) and Archaea.

Fungi a lower form of eukaryotic microorganism, may exist in the form of single-celled (yeast) and multi-celled (mold) and obtain their food by either decomposing dead organisms or by living as parasites on higher organism. These are specialized into three main types, Saprobes, Parasitic fungi and Mutlalistic fungi. 
Protozoa are another lower form of eukaryotic single-celled organism belonging to animal kingdom. They come in many different shapes and sizes ranging from an Amoeba which can change its shape to Paramecium with its fixed shape and complex structure. They live in a wide variety of moist habitats including fresh water, marine environments and the soil. Algae are lower form of photosynthetic organism exist as single-celled and multi-celled. All algae reproduce asexually and are abundant in fresh water, salt water, soil and have symbiotic association with some plants.

Viruses are smallest of the microbes, they either living or non-living organism and mostly they are pathogenic. Viruses are even smaller than bacteria and can sometimes live inside bacteria. Viruses can spread from one organism to another but it depends on the type of virus and cannot be reproduced outside.

Microbial image can be obtained from various imaging techniques, some of them are Light Microscopes Image (LMI), Scanning Electron Microscope (SEM), Transmission Electron Microscope (TEM) and Confocal Microscope (CM). The light microscope images uses light and lenses to magnify the specimen [2]. Transmission Electron Microscope (TEM) produces a twodimensional image of an ultra thin section by capturing electrons that have passed through the specimen. The light of varying intensity produced is directly proportional to the electron's Kinetic energy and used to produce the image. The TEM is useful for studying a cell's interior structure. Scanning Electron Microscope (SEM) is used to make a three-dimensional image of the specimen surface. Confocal Microscopy (CM) uses spatial filtering techniques to eliminate out-of-focus light or glare in specimens whose thickness exceeds the immediate plane of focus.

Automatic segmentation and counting of microbial organism are tedious and error prone process and moreover often these microbial images are overlapped. Therefore an efficient algorithms for the separation of overlapping bacteria and automatic counting are necessary [3].

An automatic colony counting system, which uses image processing technique to feasibly count white bacterial colonies in clear plates according to the RGB color theory is proposed in [4]. It has been proved that the method greatly improves the accuracy and efficient of the colony counting and the counting result is not affected by the shape or size of the colony. A fully automatic method which is yet cost effective microbial colony counting with both color and clear medium is developed [5]. In additional, this proposed method is software centered and can accept general digital camera images as its input. The counting process includes detecting spirals regions, identifying colonies, separating aggregated colonies, and reporting colony counts. This proposed method was robust and efficient in terms of labor and time savings.

Segmentation of bacterial images based on edge detection method was developed in [6]. They used canny edge detector on the bit-plane sliced images and then combined the edge images obtained on the high order bit plane to produce the final output. Hong et al [7] proposed a method of rapid determination of Heterotrophic bacteria in industrial cooling water. The existing colony counter devices were then developed and commercialized in the market [8]. A fully automatic colony counter with clono-counter is reported by Niyazi et al [9].

An automatic method based on statistical computation for identifying the bacteria was deviced in [10]. Bacterial cell classification using data mining techniques is employed for the classification of $\mathrm{HE}_{\mathrm{p}}-2$ cells in [11], which uses a simple set of shape features for the classification. A new 
image analysis tool to study biomass and morphotypes of three major bacterioplankton groups in an alpine lake based on geometric features is defined in Thomas Posch et al [12].

Bacterial colony counter based on two-pass iterative method is developed in [13]. In this method, a simple binarization is applied using histogram based thersholding determination. Then pattern classifier is applied to detect the shape of the bacteria, and tested for its compactness. If the compactness ratio is not within the allowable range, a watershed algorithm is applied to split the corresponding shape to disconnect the overlapping bacteria.

In this paper, we proposed a hybrid approach for automatic counting of microorganism based on morphological operation, Chan-Vese (CV) active counter and labelling method. The remaining part of the paper is organized as follows: In Section 2, the methodological details of the proposed method is given; the results and discussion are given in Section 3 and the conclusion is given in Section 4.

\section{Methodology}

Image segmentation is a process of dividing an image into multiple parts. It is used to identify the objects and its visualization. There are many different ways to segment the image such as: Thersholding based methods, region based methods, active contour-based methods etc. Global region based segmentation methods are most widely used method. Chan-Vese (CV) [14] [15] is one of the global region-based active contour segmentation methods. In this paper, we used the $\mathrm{CV}$ method to segment the microbial images for automatic counting. CV model requires to define initial mask for the segmentation, the initial mask is defined based on the width and height of the given input image. The overall block diagram of the proposed method is given in Figure 1.

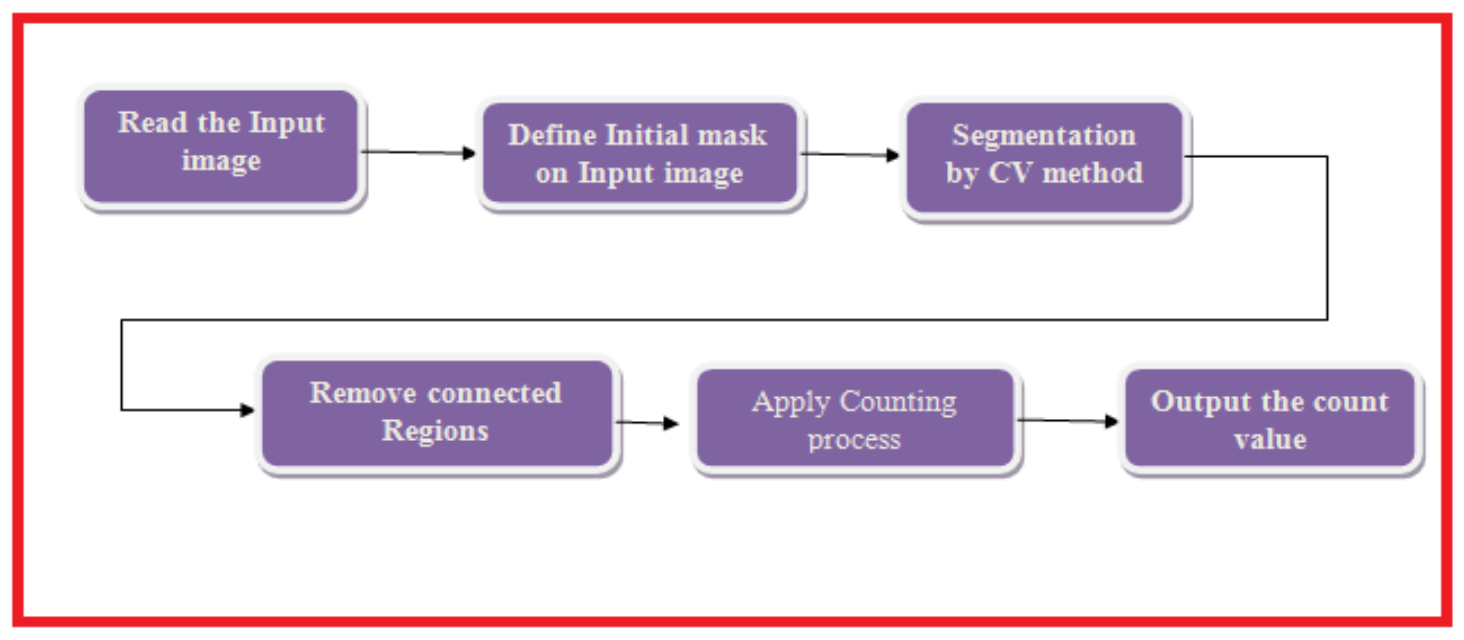

Figure 1: Block diagram of the proposed method

The CV model without edges is based on Mumford-Shah segmentation techniques. This model is described as a bimodal model, considering the image as a two distinct regions, $\mathrm{c}_{1}$ (foreground) and $c_{2}$ (background) of approximately piecewise-constant distinct intensity values. Given an image $\mathrm{u}_{0}$, a closed curve $\mathrm{C}, \mathrm{c}_{1}$ and $\mathrm{c}_{2}$ (where $\mathrm{c}_{1}$ and $\mathrm{c}_{2}$ be the average intensity of $\mathrm{u}_{0}$ at the inner 
region and outer region with respect to $\mathrm{C}$ respectively). Then, $\mathrm{CV}$ algorithm aims to minimize the energy functional $E\left(c_{1}, c_{2}, C\right)$ and is defined as:

$$
\begin{array}{r}
\left.E\left(c_{1}, c_{2}, C\right)=\mu . \text { Length }(C)+v \cdot \text { Area (inside }(C)\right) \\
+\lambda_{1} \int_{\text {inside }(C)}\left|u_{0}(x, y)-c_{1}\right|^{2} d x d y \\
+\lambda_{2} \int_{\substack{\text { ousside }(C) \\
\text { (C) }}}\left|u_{0}(x, y)-c_{2}\right|^{2} d x d y
\end{array}
$$

where, $\mu$ controls the smoothness of zero level set, $v$ increases the propagation speed, $\lambda_{1}$ and $\lambda_{2}$ controls the image data driven force inside and outside of the contour respectively and, $\mu \geq 0, v \geq 0$ and $\lambda_{1}, \lambda_{2}>0$ are fixed parameters. Then, the objective of $\mathrm{CV}$ model is to find $c_{1}, c_{2}$ and $\mathrm{C}$ such that $E\left(c_{1}, c_{2}, C\right)$ is minimized and is mathematically expressed as:

$$
\inf { }_{c_{1}, c_{2}, C} E\left(c_{1}, c_{2}, C\right)
$$

Then we convert the segmented image into binary image for automatic labelling and counting. This method uses Otsu's thersholding method to obtain the binary image. Otsu is one of the well known and widely accepted thersholding methods [16] [17] [18]. It is based on discriminant analysis to find the maximum separability of classes and is used to automatically perform the histogram shape-based image thersholding. The algorithm assumes that the image is composed of two basic classes, foreground $\left(\mathrm{C}_{0}\right)$ and background $\left(\mathrm{C}_{1}\right)$ and it construct a normalized histogram using the discrete probability density function, and is given by:

$$
p_{r}\left(r_{q}\right)=\frac{n_{q}}{n}, \quad q=0,1,2, \ldots, L-1
$$

where, $n$ is the total number of pixels in the image, $n_{q}$ is the number pixels that have intensity level $r_{q}$ and $L-1$ is the highest intensity level in that image. Initial threshold is the midpoint between the maximum and minimum intensity values in the image. If $k$ is chosen as the initial threshold, then $C_{0}$ is the set of pixels with levels $[0,1, \ldots, k-1]$ and $C_{1}$ is the set of pixels with levels $[k, k+1, \cdots, L-1]$. Otsu's method chooses the threshold value $k$ that maximizes the between-class variance $\sigma^{2}$, which is defined as:

$$
\sigma_{B}^{2}=\omega_{0}\left(\mu_{0}-\mu_{T}\right)^{2}+\omega_{1}\left(\mu_{1}-\mu_{T}\right)^{2}
$$

where

$$
\begin{gathered}
\omega_{0}=\sum_{q=0}^{k-1} p_{q}\left(r_{q}\right) \\
\omega_{1}=\sum_{q=k}^{L-1} p_{q}\left(r_{q}\right)
\end{gathered}
$$




$$
\begin{aligned}
& \mu_{0}=\sum_{q=0}^{k-1} q p_{q}\left(r_{q}\right) / \omega_{0} \\
& \mu_{1}=\sum_{q=k}^{L-1} q p_{q}\left(r_{q}\right) / \omega_{1} \\
& \mu_{T}=\sum_{q=0}^{L-1} q p_{q}\left(r_{q}\right)
\end{aligned}
$$

In general, the microbes in the microbial images are often overlapped, in order to disconnect the overlapping microbes, we applied morphological erosion operation. Morphological operations are non-linear, which are usually work based on the shape of the image feature. The shape of the image features are represented as a structuring-elements. The basic idea in binary morphological operation is to probe an image with a defined structuring element [19]. Figure 2 illustrate probing of an image with square structuring element. Most widely used structuring element are disk, square, cross etc., In our method, we used disk shape structuring element. The morphological erosion operation is defined as:

$$
A \ominus B=\left\{z \in E \mid B_{\approx} \subseteq A\right\}
$$

where $B_{z}$ is the translation of $B$ by the vector $z$.

$$
B_{z}=\{b+z \mid b \in B\}, \forall z \in E
$$

When the structuring element $B$ has a center and this center is located on the origin of $E$, then the erosion of $A$ by $B$ is expressed as:

$$
A \ominus B=\bigcap_{b \in B} A_{-b}
$$

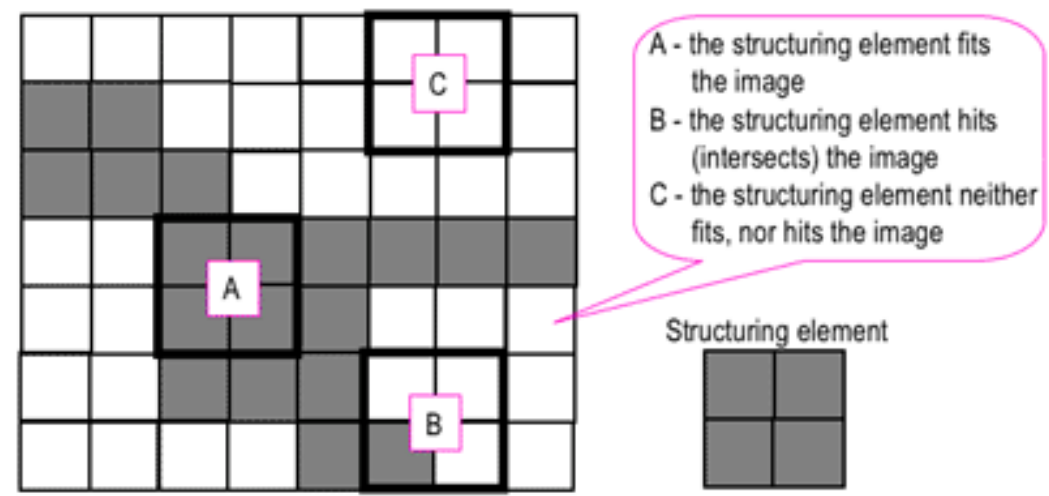

Figure 2: Probing of an image with a structuring element (white and grey pixels have zero and non-zero values, respectively).

For automatic counting, we need to fill the holes present in the eroded image. Because the presence of holes affects the labelling and counting process, we applied the holes filling and 
labelling algorithms described in [19] [20]. It's achieved using the morphological reconstruction operation as per the following equations:

$$
\begin{aligned}
& g_{m}(x, y)= \begin{cases}1, & \text { if }(x, y) \text { is on the border of } g \\
0, & \text { otherwise }\end{cases} \\
& g_{H F}=\left[R_{f} c\left(g_{m}\right)\right]^{e}
\end{aligned}
$$

where $\mathrm{g}$ is a binary image and $\mathrm{g}_{\mathrm{m}}$ is the marker images have 1 for image border and 0 for the remaining pixels, $R_{f} c$ is a reconstructed image of $g_{\mathrm{m}}$ and $\mathrm{g}_{\mathrm{HF}}$ is hole-filled binary image.

Labeling process is performed after grouping the image pixels into regions based on the 4 or 8connected neighbourhoods [20]. In connected neighbourhood, the pixels are connected to center pixel in its four neighbours left, top, right and bottom and in addition to 4-connected pixels all the 4-diagonal pixels are also included in the 8-connected neighbourhood.

The proposed methods uses 4-connected neighbourhood to identify the connected components in the hole filled image. After identification of the connected regions, we apply the region labeling algorithm [21] to assign a label number to each region for counting the number of individual microbes present in the microscopic images. The Figure 3 illustrate the process of segmenting the microscopic image by the proposed method.

In this Figure, Image (a) is the original image. The defined initial mask is shown as a red color box in Figure 3(b). The segmented image is given in Figure 3(c) and the converted binary image is given in Figure 3(d). Segmented image after morphological erosion operation is given in Figure 3(e). It can be seen from Figure 3(d) that, the bacteria are connected each other, even after the segmentation process, if we apply labelling process, it will give a count value as 2 by treating the connected bacteria as single region. Therefore we applied morphological erosion operation to disconnect these connected regions. The resulted image after applying the morphological erosion operation is given in Figure 3 (e). Summary of steps involved in the proposed method is given in Algorithm 1. 


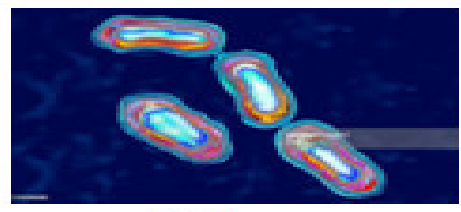

(a)

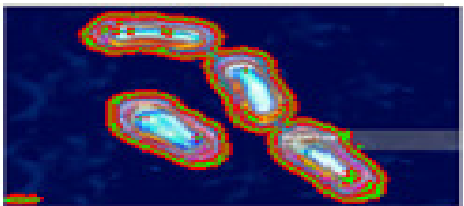

(c)

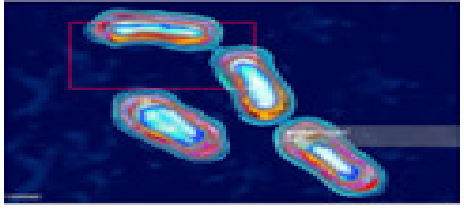

(b)

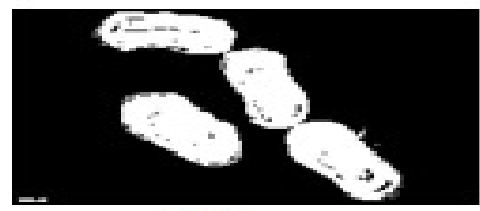

(d)

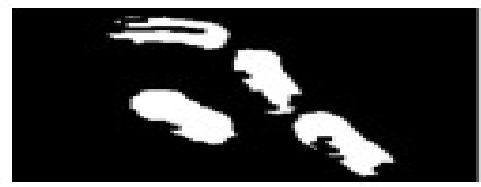

(e)

Figure 3. Segmentation of Microscopic image by proposed method; (a) Original image (b) Initial mask (c) Segmented image by CV method (d) Converted binary image (e) Segmented Image after applying morphological erosion operations.

\subsection{Algorithm 1: Segmentation and counting of microscopic Images}

1. Read the input Microscopic image.

2. If the input image is color image then convert it into gray scale image.

3. Define the initial mask and segment the image using CV method.

4. Convert the segmented image into binary image using Otsu's thersholding method.

5. Apply morphological erosion operation to disconnect the overlapped microbes.

6. Fill the holes in the binary image.

7. Apply Labeling algorithm to count the number of microbes.

8. Output the count value.

\section{RESULTS AND DISCUSSION}

To evaluate the performance of the proposed method, we have tested our method with the microscopic images obtained from the internet. A sample images along with the results obtained by the proposed and the existing method [3] are given in Figure 4. The computed count value by the propose, existing and the manual methods are given in Table 1. 


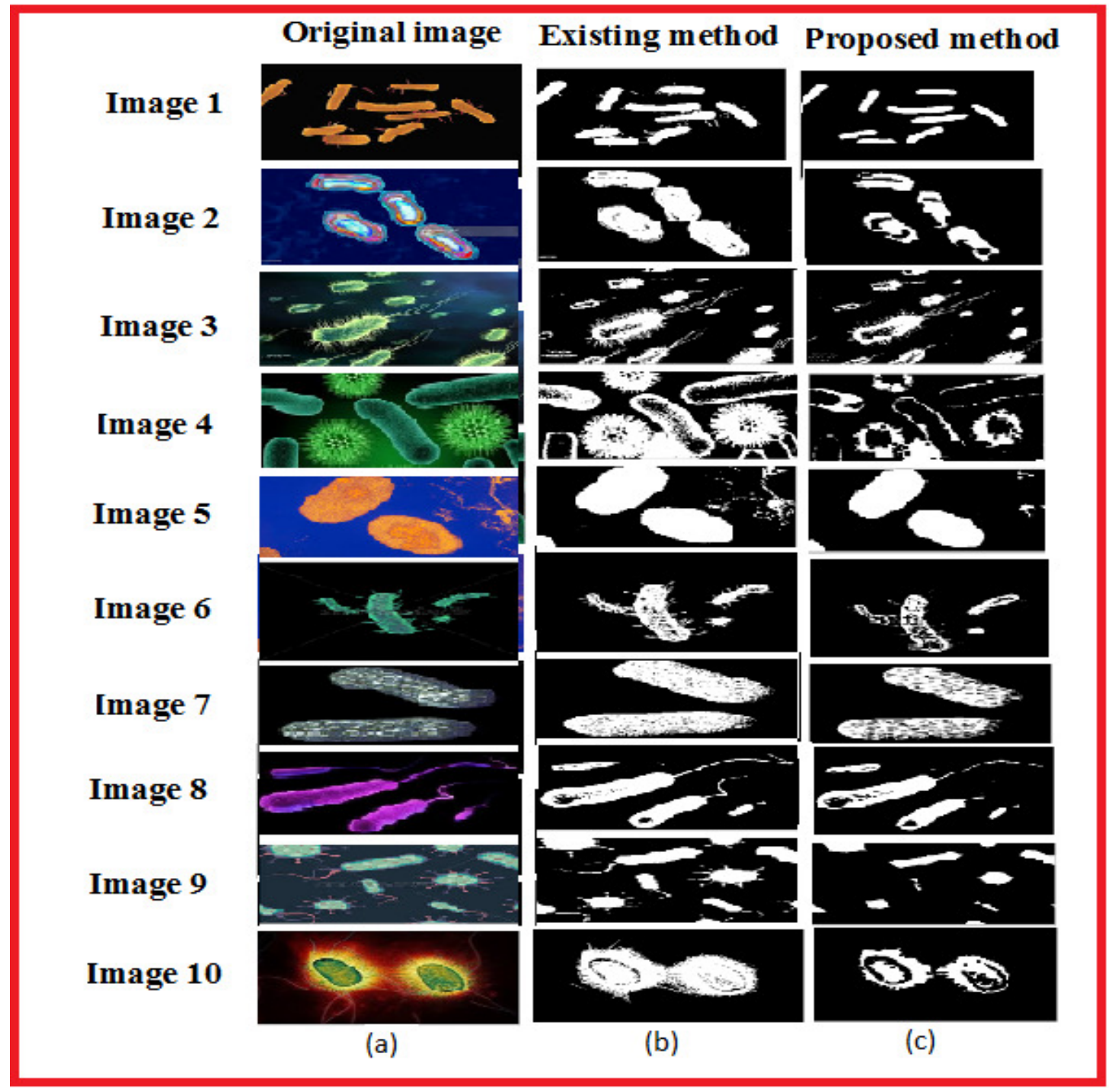

Figure 4. Segmented microscopic image by (a) Original image (b)Segmented image by Existing image (c)Segmented Image by Proposed image

In Figure 4, except in the images 7, 8 and 9, all the other images had overlapping microorganism. Since in this proposed method, we applied morphological erosion operation, which have separated these overlapping images and have produced accurate count value compared to the result obtained by the existing method and the manual count value. 
Advanced Computing: An International Journal (ACIJ), Vol.7, No.1/2, March 2016

Table 1: Microbial Count obtained by Manual count, Existing method and Proposed method

\begin{tabular}{|c|c|c|c|}
\hline Image & Manual Count & $\begin{array}{c}\text { Count value by the } \\
\text { existing method }\end{array}$ & $\begin{array}{c}\text { Count value by the } \\
\text { proposed method }\end{array}$ \\
\hline Image 1 & 9 & 7 & 9 \\
\hline Image 2 & 4 & 2 & 4 \\
\hline Image 3 & 15 & 13 & 15 \\
\hline Image 4 & 9 & 8 & 3 \\
\hline Image 5 & 3 & 5 & 4 \\
\hline Image 6 & 4 & 3 & 2 \\
\hline Image 7 & 2 & 2 & 4 \\
\hline Image 8 & 4 & 4 & 10 \\
\hline Image 9 & 10 & 9 & 2 \\
\hline Image 10 & 2 & 1 & \\
\hline
\end{tabular}

It is evident from Figure- 4 and Table- 1 that this proposed method is one of the simple and efficient methods for automatic counting of microbes in microscopic images. We have also given the manual count for quantitative comparison. This proposed methods have produced accurate counting results for all the images shown in Figure 4. Thus, this method may also find a vital role in automatic counting of microbes in microscopic images.

\section{Conclusions}

This proposed approach is a simple and efficient method for automatic segmentation and counting of microorganisms present in the microscopic images. The experimental results are compared with the manual count and also with the existing method. This proposed method have produced accurate result for all the tested images. In this work, we have taken only noise free images as input, in the future work, we have to enhance this method to count the number microorganisms present in noisy image as well as with the densed population of microorganisms in the cultured plates.

\section{REFERENCES}

[1] http:// Wikipedia.com

[2] K. Z. Mao, P. Zhao and P. Tan. "Supervised learning-based cell image segmentation for p53 immunohistochemistry",IEEE Trans Biomed Eng, vol. 53, no. 6, pp. 1153-63, 2006.

[3] P.Kalavathi and S.Naganandhini,"Automatic Segmentation and Counting of bacterial Images using CV Active Contour Model”, New Horizons Computational Intelligence and Information System, Excel Publisher, PP-182-185, New Delhi-2015.

[4] Chengcui Zhang, Wei-Bang Chen, Wen-Lin Lin, and Chi-Bang Chen, "An automated Bacterial colony counting system", pp-233-240, IEEE 2008

[5] Wei-Bang Chen \&Chengui Zhang, "An automated bacterial colony counting and classification system”, LLC 2009.

[6] P.Kalavathi, "An Efficient Edge Detection Method Based on Bit-Plane Slicing for Bacterial Images", September, Vol.6, No. (3): pgs, 315-320, 2013.

[7] Hong Men, Yujie Wu, Xiaoying Li, Zhen Kou, Shanrang Yang, "Counting Method of Heterophic Bateria Based on Image processing”, IEEE , pp-1238-1241, 2008. 
[8] J.Dahle, M.Kakar, H.B. Steen, and O.Kaalhus, "Automatic Counting of mammalian cell colonies by means of a flat bed scanner and image processing", A cytometry, vol.60, pp-182-188, 2004,.

[9] M.Niyazi, I.Niyazi, and C.Belka,"Counting Colonies of Clonogenic assays by Using Densitometric Software”, Radiation on Colony, vol.2, pp.4, 2007.

[10] Sigal Trattner and Greenspan H, "Automatic Identification of Bacterial Types Using Statistical Imaging Methods", IEEE Transactions on Medical Imaging, Vol.23 (7), pp-807-820, 2004.

[11] Petra Perner, "Classification of HEp-2 cells using Fluorescent Image Analysis and Data Mining, Medical Data Analysis", Springer Verlag, LNCS Vol.2199, pp-219-224, 2001.

[12] Thomas,P., Josef, F., Martin, P. and Michaela, M.S. "New Image Analysis Tool to Study Biomass and Morphotypes of Three Major Bacterioplankton Groups in an Alpine Lake, Acuatic Microbiol Ecology", Vol.54, pp.113-126, 2009.

[13] Huseyin Ates,Omer Nezih Gerek, "An Image-Processing Based Automated Bacteria Colony Counter", IEEE September, METU Northern Cyprus Campus , pp.14-16, 2009.

[14] K.Somasundaram and P.Kalavathi, "Skull Sipping of MRI Head Scans based on Chan-Vese Active Contour Model", International Journal of Knowledge Management \& e-learning 3(1), pp7-14, 2011.

[15] Tony Chan and Luminita Vese , “ Active Contour model Without Edges”, LNCS, Springer-Verlag. Berlin Hezidelberg, pp.141-151,1999.

[16] P.Kalavathi, "Brain Tissue Segmentation in MRI Brain Images using Otsu's Multiple Thersholding Technique",IEEE explore, pp-639-642, 2013.

[17] Otsu, N "A Threshold Selection Method from Gray-Level Histograms," IEEE Transactions on System, Man and Cybernatics Vol.9, No.1, 1979, pp.62-66.

[18] P.S. Liao, T.S. Chen and P.C. Chung, "A fast Algorithm for Multi-level Thersholding",J. Inf. Sci. Eng., Vol. 107, No. 5, pp. 713-727, 2001.

[19] Rafel C.Gonzalez, Richard Eugene woods,Steren L. Eddins., "Digital Image Processing Using MATLAB" Tata McGraw-Hill Education, 2010.

[20] L. Dong, G. Yu, P. Ogeinbona and W. li, "An Efficient Iterative Algorithm for Image Thersholding, Pattern Recognition Letter”, Vol. 29, No. 9, pp. 1311-1316, 2008..

[21] Milan Sonka, Vaclav Hlavac, Roger Boyle, "Image Processing, Analysis, and Machine Vision" , Second Edition, 2001.

\section{Authors}

Dr. P. KAlAVATHI, Associate Professor in Computer Science and Applications, Gandhigram Rural Institute - Deemed University, obtained her B.C.A degree from Mother Teresa Women's University, Kodaikanal; Post-Graduate Degree in Computer Applications (M.C.A) from GRI-DU; M.Phil., in Computer Science from Bharathidasan University, Tiruchirappalli; and Doctoral Degree in Computer Science and Applications from GRI-DU, Gandhigram. She has qualified UGC-NET for Lectureship in 2000. She has 15 years of Teaching and 8 years of Research

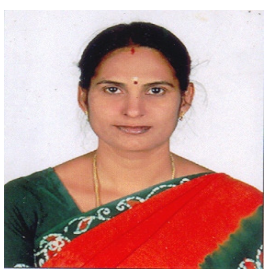
experience. Her research area focuses on Digital Image Processing and Medical Image Segmentation \& Analysis. She is also serving as a reviewer for many international conferences and for various journals in IEEE, Springer, Elsevier etc. The author is the Life Member of Indian Society for Technical Education (ISTE), New Delhi.

S.NAGANANDHINI, Completed Bachelor of Science in Computer Science in Parvathy's Arts and Science College, Madurai Kamaraj University, and Master of Computer Application in Gandhigram Rural Institute-Deemed University, Tamil Nadu. She is currently pursuing M.Phil (Computer Science) in Gandhigram Rural Institute-Deemed University, Tamil Nadu. She has published one research paper in National Conference proceedings. Her research area focus on biological image processing and analysis.

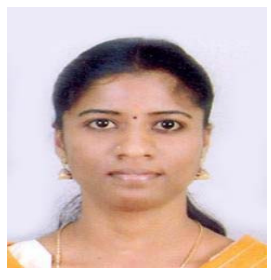

